\title{
EVIDÊNCIAS DA GLOBALIZAÇÃO NA EDUCAÇÃO CONTÁBIL: ESTUDO DAS GRADES CURRICULARES DOS CURSOS DE GRADUAÇÃO EM UNIVERSIDADES BRASILEIRAS E PORTUGUESAS
}

\author{
Edson Luiz Riccio \\ Professor Livre-Docente do Depto. de Contabilidade e Atuária - FEA/USP - Campus Capital \\ E-mail: elriccio@usp.br \\ Marici Cristine Gramacho Sakata \\ Doutorando em Controladoria e Contabilidade pela FEA/USP - Campus Capital \\ E-mail: mcsakata@usp.br
}

\section{RESUMO}

A formação dos contadores tem sido uma preocupação constante dos organismos internacionais de contabilidade como o IFAC - International Federation of Accountants e o IASB - International Accounting Standards Board, bem como da ONU - Organização das Nações Unidas, por meio de setores como o ISAR - Intergovernmental Working Group of Experts on International Standards of Accounting and Reporting/ UNCTAD - United Nations Conference on Trade and Development. Por sua vez, as instituições de ensino contábil dos diversos países são estimuladas a adequar-se às mudanças provocadas pela globalização. Assim, entende-se que quanto maior a similaridade entre o currículo adotado por um certo país e o currículo internacional, maior será a evidência de harmonização na educação contábil desse país. Para efeito de comparação, os autores consideram que a proposta feita pelo ISAR/UNCTAD/ONU de um Curriculum Mundial (CM) é a que contém a estrutura conceitual ideal ou os blocos de conhecimentos a serem ministrados pelas universidades em suas grades curriculares de Contabilidade. O estudo compara o resultado da análise de 25 Universidades Brasileiras e 25 Portuguesas em relação ao CM. Analisando-se o posicionamento em relação ao CM, percebe-se que o Bloco de Conhecimentos Administrativos e Organizacionais - tanto do Brasil como de Portugal - é o que apresentou maior aproximação. $O$ que mais se distanciou foi o bloco de conhecimentos gerais. Outra característica notada nos resultados é a quantidade ainda baixa de disciplinas de Tecnologia de Informação na maioria das grades curriculares.

Palavras-chave: Curriculum Contábil, Educação, Globalização, Curriculum Mundial, Pesquisa Comparativa.

Recebido em $12.01 .04 \cdot$ Aceito em $02.06 .04 \cdot 2^{\text {a }}$ Versão aceita em 06.07.04

* Artigo original apresentado no IX Congresso de Contabilidade, Portugal, nov. 2002. Atualizações e inclusões feitas em janeiro 2004 e julho de 2004.

\section{ABSTRACT}

Accounting education has been a constant concern for International Accounting organisms such as IFAC and IASB, as well as the UN, through sections such as ISAR/UNCTAD. Accounting education institutions from different countries, in turn, are stimulated to adapt to the changes provoked by globalization. Thus, the authors believe that, the greater the similarity between the curriculum adopted by a certain country and the international curriculum, the greater will be the evidence of harmonization in that country's accounting education. For the sake of comparison, the authors consider that the ISAR/UNCTAD/UN's proposal of a Model Curriculum (MC) contains the ideal conceptual structure or the blocks of knowledge to be supplied by the universities in their Accounting curricula. The study compares the results of an analysis of 25 Brazilian and 25 Portuguese colleges in relation to the MC. When analyzing their position in relation to the $M C$, it is noticed that the Administrative and Organizational Knowledge Block - in Brazil as well as in Portugal - presented greater proximity. The general knowledge block was most distant. Another characteristic observed in the results is the low amount of subjects on Information Technology in most of the curricula.

Keywords: Accounting Curriculum, Education, Globalization, Global Curriculum, Comparative research. 


\section{INTRODUÇÃO}

A Contabilidade permeia as sociedades nos diversos países. Seu caráter regulatório e padronizador tem sido ressaltado, ainda mais, pela globalização, como elemento fundamental para controle e avaliação do desempenho das instituições públicas e privadas.

O fenômeno da globalização tem gerado muitas alterações nas economias locais, principalmente em países emergentes. Para esses, as mudanças têm sido radicais e demandam grandes esforços nacionais para a alteração dos modelos econômicos. Entre essas, citam-se as mudanças nas leis nacionais como pré-requisito para a desregulamentação, privatização e o incremento de padrões industriais e tecnológicos internacionais para os diversos setores da economia: indústria, serviços, gestão pública e educação. As instituições educacionais estrangeiras e brasileiras iniciaram um processo de adaptação às pressões da globalização através da internacionalização dos currículos e de atividades, ou seja, diferentes estratégias estão sendo criadas para lidar com essas questões (KUMAR e USUNIER, 2001; KWIEK, 2001; WALLACE, 2003), que para muitas instituições são prioridade absoluta para continuarem viáveis. Isso inclui a internacionalização do ensino contábil. (ALTBACH e TEEICHLER, 2001; MCCABE, 2001, MENDIVIL, 2002).

Ao utilizar uma abordagem sistêmica da Contabilidade no mundo, percebe-se que a educação superior em Contabilidade é um sub-sistema do sistema mundial de contabilidade, por sua vez, formado por outros sub-sistemas: associações de profissionais, empresas, instituições públicas, órgãos regulamentadores, instituições de ensino, para citar alguns apenas, todos envolvidos na regulamentação, estruturação e propagação do conhecimento e da prática contábil no mundo.

Entre vários assuntos, a formação dos contadores tem sido uma preocupação constante dos organismos internacionais de Contabilidade como o IFAC e o IASB, bem como da ONU, por meio de setores como o ISAR/UNCTAD.

Por sua vez, as instituições de ensino contábil dos diversos países são estimuladas a adequarse às mudanças provocadas pela globalização e pela conseqüente necessidade de harmonização de conceitos e práticas. Se isso é verdade, acredita-se que deveria haver indícios de convergência das grades curriculares de Contabilidade entre os diversos países.

Na América Latina e em especial na América do Sul, os países têm características econômicas, sociais e políticas diversas, mas se assemelham no fato de serem países emergentes. Entretanto, ao longo dos últimos 40 anos, algumas ações conjuntas geraram programas de integração regional entre os países sul-americanos: a criação da ALALC - Associação Latino-Americana de Livre Comércio, em 1960, a ALADI - Associação Latino-Americana de Integração, em 1980 e posteriormente o MERCOSUL - Mercado Comum do Sul, em 1995. O MERCOSUL criou vários instrumentos de integração, inclusive na área educacional. Na maioria, são referentes a aspectos abrangentes como educação básica, e não específicos como no caso de grades curriculares de Cursos de Graduação em Contabilidade. Foi criado na América Latina a "Unión de Universidades de América Latina" e, juntamente com ela, um sistema de Informação para Educação Superior (SIESALC) para compartilhar informações e recursos educacionais entre todas as instituições da América Latina (SOSA e FEBRES, 2002).

Acredita-se que a crescente harmonização dos princípios contábeis internacionais induz à conseqüente formação de um profissional de contabilidade com características similares nos diferentes países. Para que isso aconteça, entendem os autores que a educação formal dos contadores, no mundo, deve convergir para os mesmos conjuntos de conhecimentos, harmonizados e globalizados, exceção feita ao conjunto de conhecimentos específicos de cada país, como história e cultura local. Assim, entendese que quanto maior for a similaridade entre o currículo adotado por um certo país e o currículo internacional, tanto maior será a evidência de harmonização na educação contábil desse país. Para efeito de comparação, os autores consideram que a proposta feita pelo ISAR/UNCTAD/ONU é a que contém a estrutura conceitual ideal ou os blocos de conhecimentos a serem ministrados pelas Universidades em suas grades curriculares de Contabilidade.

Neste estudo, os autores categorizam as disciplinas das grades curriculares de Contabilidade de acordo com a estrutura conceitual do ISAR/UNCTAD, ou 
seja, nos vários blocos de conhecimento. O estudo compara o resultado da análise de 25 Universidades Brasileiras e 25 Portuguesas em relação ao CM do ISAR/UNCTAD. A tabulação dos dados foi estruturada de forma a permitir a visualização dos resultados por país, universidade, bloco de conhecimento, categoria e disciplina. Além disso, os autores coletaram as declarações das Universidades quanto aos objetivos do curso de Contabilidade que oferecem, e procederam à análise a fim de detectar incidências de categorias.

\section{OBJETIVOS}

Considerando que a educação formal dos contadores parece convergir para os mesmos blocos de conhecimento, respeitadas as especificidades nacionais, os autores estabeleceram como questão da pesquisa verificar qual a proximidade dos currículos de formação contábil praticados em Portugal e no Brasil em relação ao $\mathrm{CM}$ definido pelo ISAR/ UNCTAD/ONU.

Este trabalho foi iniciado pela análise exploratória das grades curriculares de educação superior em Contabilidade praticadas por Universidades/Instituições Portuguesas e Brasileiras, comparando-as entre si e com as recomendações do ISAR/UNCTAD. $\mathrm{O}$ trabalho compara as grades curriculares a fim de identificar semelhanças e diferenças de enfoque na educação contábil entre os 2 países. A análise utilizou dados obtidos nos sites de 25 Universidades Brasileiras e 25 Universidades/Instituições de Ensino Portuguesas. Procurou-se identificar características comuns, diferenciadoras, ou que indicassem convergência em relação às recomendações do IFAC e ISAR, bem como evidências que pudessem ser relacionadas à globalização da educação contábil.

\section{REVISÃO DA LITERATURA}

O papel da grade curricular de Contabilidade na formação dos contadores.

Vários trabalhos têm sido publicados sobre questões curriculares (SMYTHE e NIKOLAI, 2002; GURGANUS, 1995), satisfação (DONELAN ET AL., 2002), habilidades e blocos de conhecimento (KIMMEL, 1995; BONK e SMITH, 1998; HERRING e WILLIAMS, 2000). O papel da grade curricular contábil na formação dos contadores, também, tem sido bastante discutido. Tais discussões envolvem características como sexo, raça e habilidades, bem como influências do meio educacional, social e político, entre outras. Além disso, uma outra discussão se torna importante nos dias de hoje: a internacionalização e a globalização de programas acadêmicos (ADHIKARI ET AL., 1999) que podem envolver conhecimento de línguas estrangeiras, cultura geral, foco, flexibilidade, entre outras características.

Porter e Carr (1999, p. 565) indicam a ocorrência de transformações nos cursos de Contabilidade no mundo anglo-americano como reação à "incapacidade dos programas existentes em preparar adequadamente os estudantes para o ambiente dinâmico e complexo dos negócios no qual entram após formados". Walsh (1998) analisa o currículo necessário para a formação do contador em função da globalização da economia. Posteriormente, Calhoun e Walsh (2000) demonstraram que existe similaridade entre os programas do IFAC (IEG9), UNCTAD, AACSB Association to Advance Collegiate Schools of Business e ACCA - Association of Chartered Certified Accountants, apesar de pequenas diferenças de conteúdo.

No entanto, nota-se a ausência, na literatura contábil internacional, de estudos abordando a educação superior em Contabilidade nos países da América Latina e entre esta e o Continente Europeu. Por outro lado, os textos que descrevem os objetivos dos cursos de Contabilidade das Universidades pesquisadas não fazem explicitamente referências sobre as intenções de direcionamento dessas grades curriculares para os padrões do IFAC e ISAR/UNCTAD.

\section{Currículo Internacional de Contabilidade}

Órgãos internacionais como IFAC, ISAR/ UNCTAD, AACSB e ACCA têm conduzido estudos objetivando a recomendação de um modelo curricular para programas acadêmicos de Contabilidade.

O ISAR/UNCTAD publicou proposta de um Currículo Modelo, também sugerido como benchmark para os países em desenvolvimento", para que estabeleçam qualificações similares aos padrões mundiais. 


\section{O MODELO DA PESQUISA}

Esse modelo de pesquisa propõe examinar as evidências referentes a: fatores em comum, fatores diferenciadores e globalização existentes nas grades curriculares de Universidades do Brasil e de Portugal. Analisa até que ponto as grades seguem as recomendações feitas pelas instituições interna- cionais. A pesquisa, também, procurou encontrar indícios que permitam entender como as Universidades preparam os futuros profissionais, ou seja, em quais habilidades necessárias para atuarem em um mundo globalizado.

Como base conceitual, este trabalho utiliza a estrutura de quatro blocos de conhecimentos definidos pelo ISAR/UNCTAD (1999):

\section{Tabela 1 - Blocos de conhecimentos definidos pelo ISAR/UNCTAD (1999)}

\begin{abstract}
1. Conhecimentos administrativos e organizacionais

1.1 Economia.

1.2 Métodos Quantitativos e estatística para administração. 1.3 Políticas gerais administrativas, estruturas básicas organizacionais.

1.4 Funções e práticas gerenciais, comportamento organizacional, a função do marketing em administração e princípios de negócios internacionais.

1.5 Módulo de gestão e estratégia organizacional.
\end{abstract}

\section{Tecnologia de} Informação

2.1 Tecnologia de Informação.

2.1.1 Tecnologia de Informação (TI) conceitos para sistemas administrativos.

2.1.2 Controle interno - sistemas informatizados de gestão. 2.1.3 Desenvolvimento de padrões e práticas para a administração de sistemas.

2.1.4 Gestão, implementação e uso de TI.

2.1.5 Gestão da segurança em informação.

2.1.6 Inteligência Artificial, expert systems, fuzzy logic, etc. 2.1.7 Comércio Eletrônico.

\section{Conhecimentos de Conta-} bilidade e assuntos afins

3.1 Contabilidade básica e preparação de relatórios financeiros, a profissão contábil padrões contábeis internacionais.

3.2 Práticas contábeis e financeiras avançadas.

3.3 Princípios de relatórios financeiros avançados.

3.4 Contabilidade Gerencial. 3.5 Contabilidade Gerencial Informação para planejamento, tomada de decisão e controle. 3.6 Tributação.

3.7 Legislação comercial. 3.8 Fundamentos de Auditoria. 3.9 Auditoria: conceitos avançados.

3.10 Finanças e gestão financeira.

\section{Conhecimentos} Gerais

4.1 História e religião.

4.2Comportamento humano/ Psicologia.

4.3 Economia local

4.4 Metodologia de pesquisa. 4.5 Artes e Literatura.

4.6 Ética.

4.7 Filosofia.

4.8 Comunicação oral.

4.9 Línguas.

4.10 Experiência profissional/ Estágio.
Os blocos 1, 2 e 3, são exatamente os sugeridos no texto do ISAR/UNCTAD(1999). O bloco 4 Conhecimentos Gerais - foi adaptado pelos autores a partir do texto original apresentado pelo documento a fim de formar categorias de conhecimento que possibilitassem comparação e análise.

$O$ estudo categoriza as disciplinas das grades curriculares de cada Universidade de acordo com a estrutura conceitual acima. Dessa forma, podese efetuar uma comparação dos resultados por país, universidade, bloco de conhecimento, categoria e disciplina.

Além disso, os autores acessaram as declarações das Universidades que contêm os OBJETIVOS dos Cursos de Contabilidade. A partir da análise de conteúdo dos textos, os autores destacaram palavras e expressões que demonstram o tipo de profissional que a Universidade se propõe a formar.
As palavras e expressões foram agrupadas segundo a freqüência de aparecimento nos textos.

Ambas as análises permitem o entendimento dos objetivos reais e declarados de cada Universidade para o curso de Ciências Contábeis. Os resultados apóiam as discussões acerca da globalização das grades curriculares do Brasil e de Portugal e de pesquisa anterior envolvendo os demais países da América do Sul.

\section{POPULAÇÃO}

A população deste estudo é formada por todas as Universidades dos Países em questão, que segundo Forster (2004), em seu Banco de Dados sobre Universidades no Mundo, totalizam 142 Universidades no Brasil e 60 Universidades e Institutos em Portugal. Embora possa haver alguma variação, tomou-se por base esses dados. 


\section{AMOSTRA E COLETA DE DADOS}

Para a pesquisa, a amostra compreendeu 25 cursos de Contabilidade em Universidades e Faculdades do Brasil e 25 cursos de Contabilidade ou área relacionada em Universidades e Instituições de Ensino de Portugal. Como em Riccio e Sakata (2002), os detalhes das grades curriculares foram levanta- dos por meio de acesso ao Website, além do contato com a Universidade por meio de email para a complementação de informações. A escolha das Universidades foi baseada no conhecimento dos autores e informações obtidas junto a professores e estudantes dos países pesquisados.

A tabela abaixo apresenta as Universidades pesquisadas:

\section{Tabela 2 - Amostra de Universidades do Brasil e Portugal}

\section{BRASIL}

1) Universidade Federal de Santa Maria

2) Universidade Federal de Minas Gerais

3) Universidade Federal de Santa Catarina

4) Universidade Federal do Maranhão

5) Universidade Federal do Sergipe

6) Universidade Federal do Ceará

7) Universidade Federal de Uberlândia

8) Universidade do Vale do Rio dos Sinos

9) Faculdades Associadas de São Paulo

10) Pontifícia Universidade Católica - Minas Gerais

11) Pontifícia Universidade Católica - Paraná

12) Pontifícia Universidade Católica - Rio Grande do Sul

13) Universidade Federal de Viçosa

14) Universidade Federal do Rio Grande do Sul

15) Universidade Federal do Rio de Janeiro

16) Universidade de São Paulo

17) Universidade de Brasília

18) Universidade Mackenzie

19) Universidade Federal de Pernambuco

20) Universidade Estadual do Rio de Janeiro

21) Universidade Estadual da Paraíba

22) Universidade Federal do Paraná

23) Universidade Estadual do Oeste do Paraná

24) Universidade Federal do Mato Grosso

25) Fundação Santo André

\section{PORTUGAL}
1) Instituto Politécnico do Porto
2) Instituto Sup. de Ciências do Trabalho e da Empresa
3) Instituto Superior Politécnico de Gaya
4) Universidade de Aveiro
5) Universidade Fernando Pessoa
6) Universidade dos Açores
7) Instituto Superior de Contabilidade e Administração
8) Instituto Politécnico de Tomar
9) Instituto Superior de Gestão
10) Universidade Católica Portuguesa
11) Universidade da Beira Interior
12) Faculdade de Economia do Porto
13) Universidade Independente
14) Universidade Moderna - Setúbal
15) Universidade Lusíada de Lisboa
16) Universidade Portucalense
17) Universidade Técnica de Lisboa
18) Universidade Autônoma de Lisboa
19) Universidade do Algarve
20) Universidade Internacional
21) Instituto Superior de Adm., Comunic. e Empresa
22) Universidade da Madeira
23) Universidade de Coimbra
24) Universidade de Évora
25) Universidade Lusófona

\section{RESULTADOS E CONCLUSÕES}

A média de disciplinas oferecidas nas 25 Universidades Brasileiras (média de 42 disciplinas) e 25 Universidades Portuguesas (média de 45 disciplinas) é próxima, o que permitiu as comparações. No entanto, não se pode considerar esses valores isoladamente, uma vez que se faz necessário estudar todo o conteúdo programático da disciplina e não apenas a nomenclatura. Porém, tratando-se de uma pesquisa exploratória, tais resultados podem oferecer indícios sobre as diferenças na Educação Contábil entre os países.

A média simples das disciplinas por blocos de conhecimento é mostrada a seguir:

As disciplinas de Contabilidade, nas Universidades
Brasileiras constituem praticamente $50 \%$ do curso. De forma agregada, tem-se uma média de 25 disciplinas contábeis em cada curso (ver Anexo 1). Os gráficos apresentados, a seguir, mostram a distribuição das disciplinas por curso. Embora existam variações - como o número de horas aula - nenhuma das 25 Universidades apresentou grandes variações diante das demais. $O$ segundo bloco de disciplinas mais oferecidas é o de conhecimentos organizacionais, que engloba as disciplinas quantitativas, econômicas e administrativas, sendo que o número de disciplinas varia de 5 a 10 por curso. As disciplinas de conhecimentos gerais, em menor quantidade, aparecem em terceiro lugar em quantidade de oferecimentos. As diferenças encontradas entre os cursos foram maiores: alguns oferecem apenas 
uma disciplina, enquanto outros chegam a oferecer até 12 disciplinas. Deve-se notar que dentre essas disciplinas se encontram as de Religião, e Universidades confessionais oferecem um maior leque de disciplinas dessa ordem, como, por exemplo, as Pontifícias Universidades Católicas, presentes em diversos Estados Brasileiros. O aluno pode cursar disciplinas optativas, tanto dentro da escola como em outras escolas de diferentes áreas. As disciplinas de Tecnologia de Informação são pouco oferecidas. A maioria oferece de 1 a 2 , em geral disciplinas básicas de Introdução à Informática, não abrangendo Sistemas Empresariais, Sistemas Contábeis, Integrados ou Auditoria de Sistemas.

Seguindo a mesma metodologia de análise usada para as Universidades Brasileiras, o resultado mostrou que a maior concentração de disciplinas nas Universidades Portuguesas, diferente do Brasil, se dá nas disciplinas de Conhecimentos Organizacionais, embora a diferença seja pequena, seguida pelas disciplinas de Contabilidade. São oferecidas, em média, de 15 a 20 disciplinas de Conhecimentos Organizacionais embora em algumas Universidades sejam oferecidas mais de 25 disciplinas de Contabilidade.

As disciplinas de Conhecimentos Gerais não são homogêneas em quantidade variando de 0 a 11 disciplinas. Quanto às disciplinas optativas, essas são oferecidas sem homogeneidade entre as Universidades Portuguesas.

\section{Comparação: Brasil e Portugal em relação ao CM do ISAR/UNCTAD}

A comparação foi realizada pela quantificação dos blocos de conhecimento de acordo com a quantidade de desdobramentos do primeiro nível de abertura. As disciplinas das 50 Universidades (25 brasileiras e 25 portuguesas) foram classificadas de acordo com o padrão do ISAR/UNCTAD e receberam o valor "1" quando possuíam correspondência com o ISAR/UNCTAD e "0" quando não possuíam.

Dessa forma, tem-se um total de 26 "disciplinaspadrão", sendo 5 de conhecimentos administrativos e organizacionais, 1 de Tecnologia de Informação, 10 de Contabilidade e assuntos afins e 10 de Conhecimentos Gerais. (ver Tabela 1)

Considerando o total de módulos de disciplinas presentes no documento do ISAR/UNCTAD, tem-se que o Brasil se aproxima das disciplinas sugeridas pelo Modelo. Nos gráficos 1, 23 e 4, o eixo Y corresponde à distância, em número de disciplinas, das grades curriculares das Universidades em relação ao documento do ISAR/ UNCTAD, e o eixo $X$ corresponde a cada uma das 25 Universidades de cada País analisado.

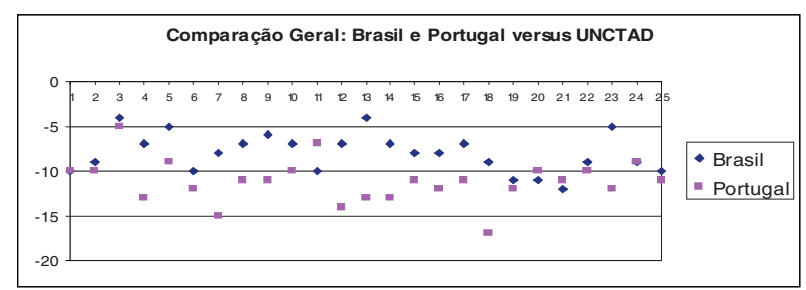

\section{Gráfico 1 - Comparação Brasil e Portugal versus UNCTAD}

Abrindo a comparação por Bloco de conhecimentos, o gráfico abaixo mostra que quanto às disciplinas de Contabilidade, o Brasil também está mais próximo do documento da UNCTAD.

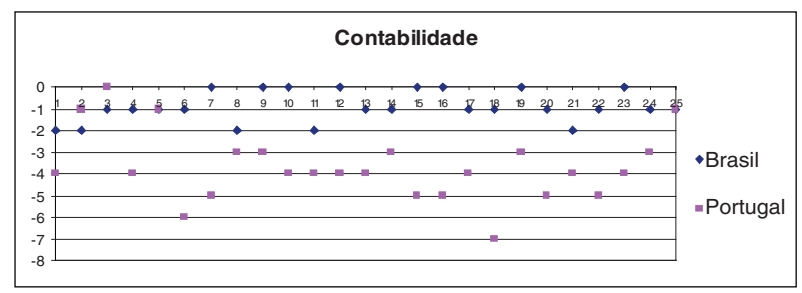

Gráfico 2 - Comparação: Brasil e Portugal versus ISAR/UNCTAD/Bloco de Contabilidade

Quanto ao bloco de Conhecimentos Organizacionais e Administrativos, Portugal encontra-se mais próximo ao sugerido no documento da UNCTAD.

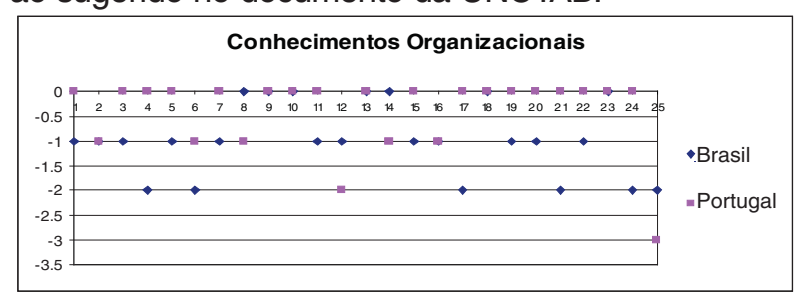

Gráfico 3 - Comparação: Brasil e Portugal versus ISAR/UNCTAD/Bloco de Conhecimentos Organizacionais

Quanto ao bloco de Conhecimentos Gerais, ambos os países possuem grandes variações em comparação ao documento da UNCTAD, ficando distantes das disciplinas sugeridas pela UNCTAD.

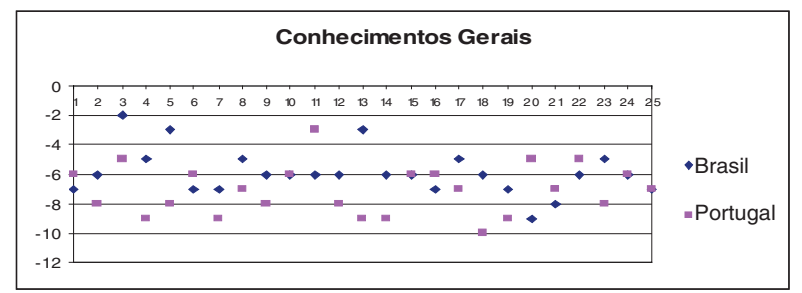

Gráfico 4 - Comparação: Brasil e Portugal versus ISAR/UNCTAD/Bloco de Contabilidade 
A tabela 3 apresenta o resumo dos dados encontrados:

Tabela 3 - Porcentagem de disciplinas por Bloco de Conhecimentos

\begin{tabular}{l|c|c|} 
& Universidades Brasileiras $(\mathbf{n = 2 5})$ & Universidades e Instituições Portuguesas $(\mathbf{n}=\mathbf{2 5})$ \\
\hline Conhecimentos adm. e organizacionais & $22 \%$ & $41 \%$ \\
Tecnologia de Informação & $3 \%$ & $5 \%$ \\
Contabilidade e conhecimentos afins & $56 \%$ & $39 \%$ \\
Conhecimentos Gerais & $14 \%$ & $9 \%$ \\
Disciplinas Optativas & $5 \%$ & $6 \%$ \\
\hline
\end{tabular}

Os autores entendem que a existência de certas disciplinas no currículo representa indícios de globalização, como por exemplo, línguas estrangeiras e temas internacionais.

Com relação às línguas estrangeiras em Portugal, foram encontradas 27 disciplinas (Inglês e Francês). No Brasil foram encontradas 4 (Inglês).

Com relação a temas internacionais foram encontradas 17 disciplinas em Portugal e 8 no Brasil, conforme lista de quantidade e disciplinas a seguir:

- Brasil: (4) Contabilidade Internacional; (3) Economia Internacional e (1) Marketing Internacional.

- Portugal: (4) Economia Internacional; (2) Direito Econômico Internacional; (2) Gestão Financeira Internacional; (2) Negócios Internacionais; (1) Teoria e Sistemas das Relações Internacionais; (1) Contratos Internacionais; (1) Marketing Internacional; (1) Cooperação
Internacional; (1) Organizações Internacionais; (1) Finanças Internacionais; (1) Contabilidade Internacional.

\section{Análise dos objetivos declarados dos Cursos de Ciências Contábeis}

Os autores analisaram os textos contendo os objetivos dos cursos declarados pelas Universidades sobre a formação do profissional de contabilidade. Do total de 50 Universidades 14 não apresentavam os objetivos do curso em seu site. Procurou-se categorizar as expressões que mais apareceram nos "Objetivos do Curso". Em Portugal, os objetivos declarados com maior freqüência são: proporcionar uma visão ampla e interdisciplinar da Contabilidade e do Mercado; em seguida a necessidade de unir a teoria à prática, e em terceiro lugar fornecer conhecimentos em gestão e controle. A categoria correspondente à Globalização é citada em 6 sites de Universidades/Instituições de Ensino Portuguesas. A tabela 4 abaixo mostra as categorias que mais se destacaram:

\section{Tabela 4 - Categorização dos objetivos das Universidades e Instituições de Ensino portuguesas $(n=18)$}

\begin{tabular}{l|l|l|c}
\hline Categorias & Freqüência & Categorias (cont) & Freqüência \\
Visão ampla \& Interdisciplinar & 8 & Competitividade e Estratégia & 2 \\
\hline Teoria e Prática & 7 & Profissional preparado e confiante & 2 \\
\hline Gestão e Controle & 6 & Conhecedor de Sistema de Informação & 1 \\
\hline Preparação para a Globalização/ & & & \\
Modernidade & 6 & Atividades para Setor Público \& Privado & 1 \\
\hline Finanças & 4 & Fiscal e Tributária & 1 \\
\hline Competências próprias/específicas & 4 & Inserção Profissional & 1 \\
\hline Comércio exterior & 4 & Voltado à sociedade e a investidores & 1 \\
\hline Auditoria & 2 & Consultor & 1 \\
\hline Pequenas e Médias Empresas & 2 & &
\end{tabular}


No Brasil, os objetivos declarados, com maior freqüência, são: oferecer conhecimentos em gestão e controle e, em seguida, necessidade de conhecer sistemas de informação e de finanças. Docência e pesquisa aparecem em igual freqüência. A categoria correspondente à Globalização aparece logo a seguir, em 5 sites de Universidades Brasileiras. A tabela 5 abaixo mostra as categorias que mais se destacaram:

\section{Tabela 5 - Categorização dos objetivos das Universidades e Instituições de Ensino Brasileiras $(n=18)$}

\begin{tabular}{l|c|l} 
Categorias & Freqüência & Categorias (cont) \\
Gestão e Controle & 12 & Atividades para Setor Público \& Privado \\
\hline Conhecedor de Sistema de Informação & 6 & Profissional Autônomo \\
\hline Finanças & 6 & Visão ampla \& Interdisciplinar \\
\hline Docência e Pesquisa & 6 & Auditoria \\
\hline Ética & 5 & Competências próprias/específicas \\
\hline Preparado para a Globalização/Modernidade & 5 & Consultor \\
\hline Voltado à sociedade e a investidores & 5 & Profissional preparado e confiante \\
\hline Inserção Profissional & 4 & Teoria e Prática
\end{tabular}

\section{DISCUSSÃO FINAL}

Embora existam certas limitações no estudo, pode-se notar que algumas indicações de globalização no currículo são evidentes, especialmente pela análise dos objetivos de curso declarados nos sites. No entanto, na comparação com o currículo modelo sugerido pelo ISAR/UNCTAD, a variação ainda é grande. No geral, as Universidades Brasileiras estão mais próximas do modelo que as Portuguesas (principalmente em relação às disciplinas de Contabilidade), mas nenhuma tanto em Portugal quanto no Brasil informa, ao menos no site, estar seguindo um padrão internacional. Algumas Universidades/Instituições, em particular, mostram-se muito afastadas desse modelo. Analisando essa aproximação ao modelo do ISAR/UNCTAD, percebe-se que o Bloco de Conhecimentos Administrativos e Organizacionais, tanto no Brasil como em Portugal é o que apresentou maior aproximação. $O$ que mais se distanciou foi o bloco de Conhecimentos Gerais. Outra característica notada nos resultados é a baixa quantidade de disciplinas de Tecnologia de Informação presente na maioria das grades curriculares. Foram encontradas 27 disciplinas de línguas estrangeiras nas Universidades Portuguesas como Inglês e Francês - pode-se notar indícios de globalização refletindo provavelmente o resultado da União Européia. Outra evidência esperada da globalização do currículo contábil seria a presença de disciplinas relacionadas a assuntos internacionais. De um total de 1906 disciplinas obrigatórias na América do Sul, apenas 3 referem-se a Contabilidade Internacional, 10 a Negócios Internacionais e uma a Economia Internacional. Os autores consideram um índice por demais baixo, uma vez que o estudo da Contabilidade Internacional poderia ser um dos indicativos de globalização.

\section{LIMITAÇÕES E PESQUISAS FUTURAS}

Algumas limitações deste estudo podem ser destacadas. Em primeiro lugar, o estudo não considera as diferenças entre Universidades Públicas e Privadas. Em segundo, a análise das grades curriculares, neste estudo, é exploratória e não tem a intenção de concluir aspectos referentes à qualidade do ensino de Contabilidade em qualquer uma das Universidades citadas. Outros estudos devem ser desenvolvidos a fim de fornecer considerações mais aprofundadas sobre outros fatores, tais como aspectos culturais, políticos e socioeconômicos. 


\section{REFERÊNCIAS BIBLIOGRÁFICAS}

ALTBACH, P.; TEICHLER, U. Internationalization and Exchanges in a Globalized University, Journal of Studies in International Education, Vol.5, No.1, Spring: 5-25, 2001.

ADHIKARI, A.; FLANIGAN, M.; TONDKAR, R. H. A survey of international accounting education in the US and some other countries, Journal of Accounting Education, Volume 17, Issues 2-3, Spring-Summer: 175-189, 1999.

BONK, C.; SMITH, G. S. Alternative instructional strategies for creative and critical thinking in the accounting curriculum, Journal of Accounting Education, Volume 16, Issue 2, August: 261-293, 1998.

CALHOUN, C.; WALSH, M. UNF's Accounting Curriculum versus the United Nation's Global Curriculum and the ACCA Curriculum: a Comparison. Accounting \& Business, 2000.

DONELAN J. G.; PHILIPICH, K. L. Meeting the 150-hour requirement: the impact of curriculum choice on satisfaction, Journal of Accounting Education, Volume 20, Issue 2, Spring: 105-121, 2002.

FÖRSTER, K. Universities Worldwide, University of Innsbruck, Department of Geography, 2004. Disponível em: <http:// geowww.uibk.ac.at/univ/world.html>. Acesso em: 10 jan. 2004.

Guideline on National Requirements for the Qualification of Professional Accountants, United Nations Conference on Trade and Development - UNCTAD, New York/Geneve, 1999.

GURGANUS, F.E.; DOTY, E.A.; HAGLER, J.L. Ecu curriculum responds to the need of multiple constituents. Journal of Accounting Education, East Carolina, v.13, n.2, p.223-240, 1995, 1995.

HERRING III H. C.; WILLIAMS, J. R. The role of objectives in curriculum development, Journal of Accounting Education, Volume 18, Issue 1, 1 March: 1-14, 2000.

JONHSON, G. Preparing students to thrive in the new economy: do accounting curricula reflect IFAC education recommendations? Proceedings: $13^{\text {th }}$ Asian Pacific Conference on International Accounting Issues, Rio de Janeiro, Brazil, 2001.
KIMMEL, P. A framework for incorporating critical thinking into accounting education, Journal of Accounting Education, Volume 13, Issue 3, Summer: 299-318, 1995.

KUMAR, R.; USUNIER, J. Management Education in a Globalizing World Lessons from the French Experience, Management Learning, Sage Publications, London Vol. 32(3): 363-391, 2001.

KWIEK, M. The Internationalization and Globalization in Central and East European Higher Education, Society for Research in Higher Education International News, $\mathrm{N}^{\circ} 47$, November: 3-5, 2001.

MCCABE, L. Globalization and Internationalization: The Impact of Education Abraod Programs, Journal of Studies in International Education, Vol. 5 No. 2, Summer, 2001.

MENDIVIL, J. The new providers of higher education, Higher Education Policy, Volume 15, Issue 4 December, Pages 353-364, 2002.

PORTER, B.; CARR, S. From Strategic Plan to Practical Realities: Developing and Implementing a Zero-Based Accounting Curriculum, Issues in Accounting Education, Vol. 14, No. 4, November, 1999.

RICCIO, E.L., SAKATA, M.G. Effects on Globalization in Accounting Education in South American Universities, $14^{\text {th }}$ Asian Pacific Conference, 2002.

SOSA, J.; FEBRES, S. Academic freedom and social responsibility: the role of university organisations and possible instruments for international monitoring, Higher Education Policy, Volume 15, Issue 4 December, Pages 385-390, 2002.

SMYTHE, M.; NIKOLAI, L. A. A thematic analysis of oral communication concerns with implications for curriculum design, Journal of Accounting Education, Volume 20, Issue 3, Summer: 163-181, 2002.

WALLACE, M. Managing the Unmanageable?: Coping with Complex Educational Change. Educational Management \& Administration, Vol 31 (1), p. 9-29, 2003.

NOTA:

Endereço dos autores:

EDSON LUIZ RICCIO

MARICI CRISTINE GRAMACHO SAKATA

Universidade de São Paulo

Faculdade de Economia, Adm. e Contabilidade

Av. Prof. Luciano Gualberto, 908 - Butantã

São Paulo - SP - 05508-900 


\section{UNIVERSIDADES DO BRASIL}

\begin{tabular}{|c|c|c|c|c|c|c|c|c|c|c|c|c|c|c|c|c|c|c|c|c|c|c|c|c|c|c|}
\hline Universidade/Faculdade & 1 & 2 & 3 & 4 & 5 & 6 & 7 & 8 & 9 & 10 & 11 & 12 & 13 & 14 & 15 & 16 & 17 & 18 & 19 & 20 & 21 & 22 & 23 & 24 & 25 & Méd \\
\hline Contabilidade & 26 & 31 & 25 & 23 & 29 & 29 & 28 & 21 & 27 & 25 & 26 & 25 & 20 & 27 & 19 & 20 & 30 & 29 & 25 & 41 & 18 & 17 & 18 & 16 & 19 & 24.6 \\
\hline Conhecimentos & & & & & & & & & & & & & & & & & & & & & & & & & & \\
\hline Organizacionais & 11 & 7 & 13 & 8 & 5 & 5 & 12 & 7 & 18 & 11 & 14 & 8 & 5 & 11 & 8 & 13 & 6 & 21 & 9 & 16 & 5 & 5 & 5 & 5 & 11 & 9.56 \\
\hline Conhecimentos Gerais & 5 & 7 & 11 & 8 & 3 & 5 & 7 & 6 & 6 & 10 & 12 & 7 & 5 & 1 & 3 & 4 & 7 & 7 & 3 & 2 & 4 & 5 & 7 & 6 & 6 & 5.88 \\
\hline Disc. Optativas & 6 & 2 & 3 & 8 & 4 & 0 & 0 & 2 & 0 & 0 & 0 & 0 & 11 & 6 & 8 & 8 & 0 & 1 & 1 & 0 & 2 & 0 & 0 & 3 & 0 & 2.6 \\
\hline Tecnologia de Informação & 2 & 1 & 2 & 2 & 1 & 1 & 1 & 2 & 5 & 1 & 0 & 1 & 1 & 1 & 1 & 2 & 2 & 1 & 1 & 2 & 1 & 1 & 1 & 1 & 1 & 1.4 \\
\hline TOTAL & 2 & 48 & 54 & 49 & 42 & 40 & 48 & 38 & 56 & 47 & 52 & 41 & 42 & 46 & 39 & 47 & 45 & 59 & 39 & 61 & 30 & 28 & 31 & 31 & 37 & 42.1 \\
\hline
\end{tabular}

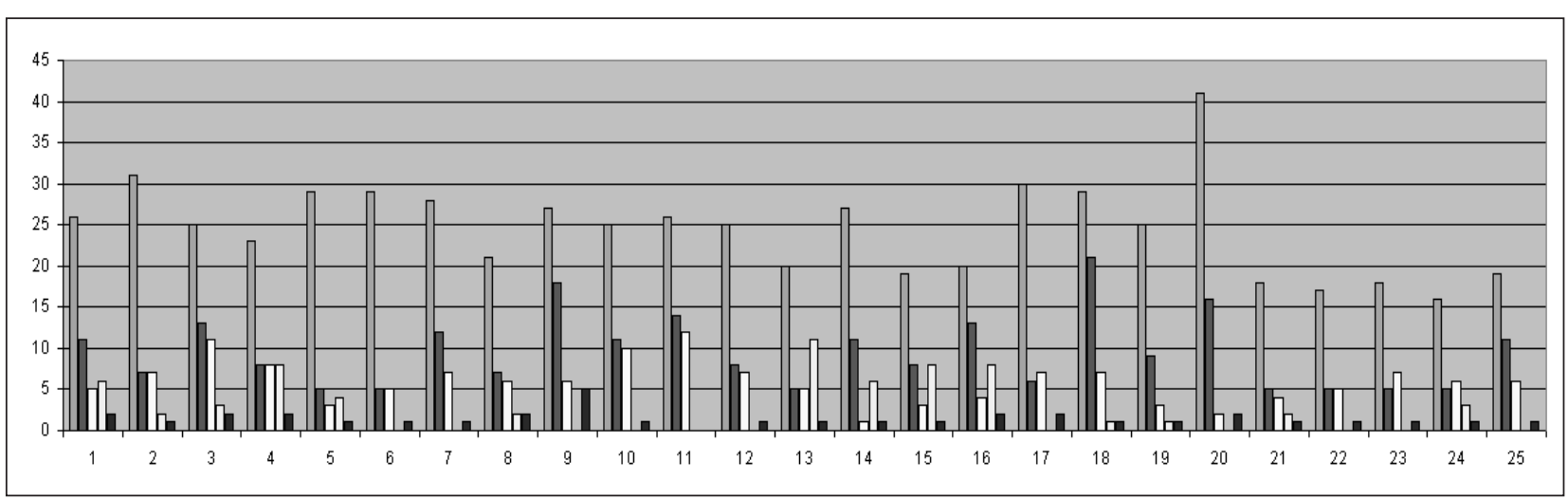

\section{UNIVERSIDADES E INSTITUTOS DE PORTUGAL}

\begin{tabular}{|c|c|c|c|c|c|c|c|c|c|c|c|c|c|c|c|c|c|c|c|c|c|c|c|c|c|c|}
\hline Universidade/Faculdade & 1 & 2 & 3 & 4 & 5 & 6 & 7 & 8 & 9 & 10 & 11 & 12 & 13 & 14 & 15 & 16 & 17 & 18 & 19 & 20 & 21 & 22 & 23 & 24 & 25 & Méd \\
\hline Contabilidade & 32 & 17 & 23 & 29 & 15 & 11 & 22 & 28 & 12 & 13 & 18 & 19 & 20 & 21 & 13 & 16 & 8 & 16 & 16 & 13 & 11 & 11 & 12 & 20 & 30 & 17.8 \\
\hline \multicolumn{27}{|l|}{ Conhecimentos } \\
\hline Organizacionais & 15 & 18 & 13 & 10 & 23 & 22 & 14 & 14 & 19 & 23 & 34 & 25 & 26 & 28 & 22 & 18 & 17 & 23 & 8 & 19 & 14 & 19 & 20 & 18 & 6 & 18.7 \\
\hline Conhecimentos Gerais & 10 & 1 & 1 & 4 & 11 & 1 & 5 & 3 & 5 & 9 & 2 & 5 & 2 & 8 & 4 & 3 & 0 & 1 & 6 & 4 & 7 & 3 & 2 & 4 & 6 & 4.28 \\
\hline Disc. Optativas & 0 & 1 & 0 & 8 & 1 & 11 & 0 & 0 & 2 & 0 & 0 & 0 & 0 & 4 & 0 & 0 & 14 & 2 & 2 & 2 & 0 & 6 & 2 & 2 & 0 & 2.28 \\
\hline Tecnologia de Informação & 4 & 2 & 4 & 1 & 2 & 0 & 1 & 4 & 2 & 2 & 3 & 3 & 3 & 3 & 2 & 2 & 2 & 1 & 2 & 3 & 3 & 1 & 2 & 2 & 2 & 2.24 \\
\hline TOTAL & 61 & 39 & 41 & 52 & 52 & 45 & 42 & 49 & 40 & 47 & 57 & 52 & 51 & 64 & 41 & 39 & 41 & 43 & 34 & 41 & 35 & 40 & 38 & 46 & 44 & 45.4 \\
\hline
\end{tabular}

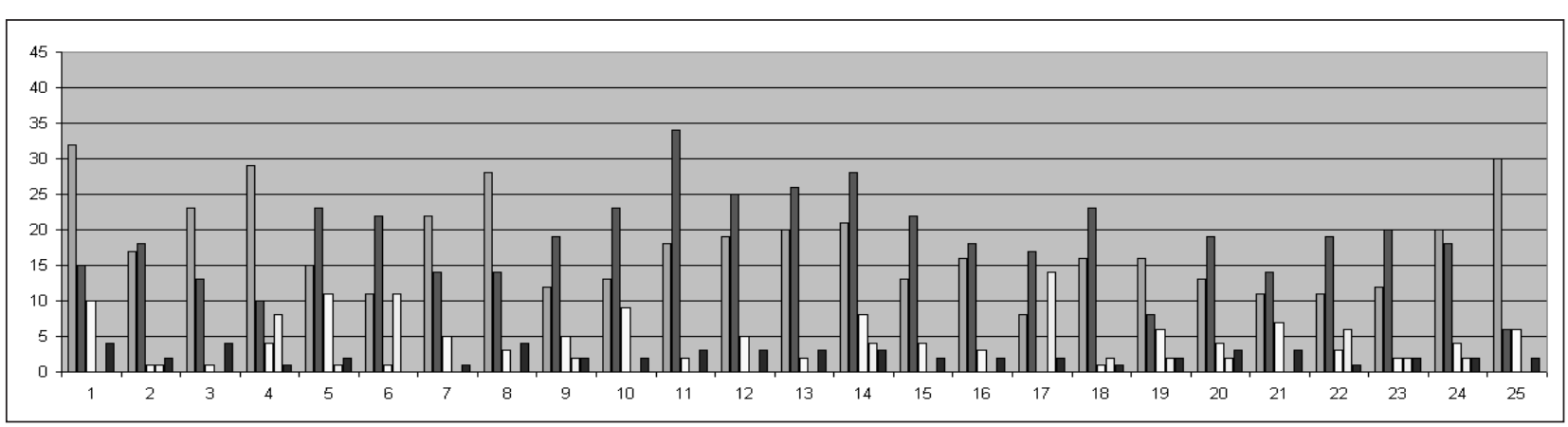

